\title{
Conformational Stability Study of a Therapeutic Peptide Plectasin Using Molecular Dynamics Simulations in Combination with NMR
}

Indrakumar, Sowmya; Zalar, Matja; Pohl, Christin; Nørgaard, Allan; Streicher, Werner; Harris, Pernille; Golovanov, Alexander P.; Peters, Günther H.J.

Published in:

Journal of Physical Chemistry Part B: Condensed Matter, Materials, Surfaces, Interfaces \& Biophysical

Link to article, DOI:

10.1021/acs.jpcb.9b02370

Publication date:

2019

Document Version

Peer reviewed version

Link back to DTU Orbit

Citation (APA):

Indrakumar, S., Zalar, M., Pohl, C., Nørgaard, A., Streicher, W., Harris, P., Golovanov, A. P., \& Peters, G. H. J. (2019). Conformational Stability Study of a Therapeutic Peptide Plectasin Using Molecular Dynamics

Simulations in Combination with NMR. Journal of Physical Chemistry Part B: Condensed Matter, Materials, Surfaces, Interfaces \& Biophysical, 123(23), 4867-4877. https://doi.org/10.1021/acs.jpcb.9b02370

\section{General rights}

Copyright and moral rights for the publications made accessible in the public portal are retained by the authors and/or other copyright owners and it is a condition of accessing publications that users recognise and abide by the legal requirements associated with these rights.

- Users may download and print one copy of any publication from the public portal for the purpose of private study or research.

- You may not further distribute the material or use it for any profit-making activity or commercial gain

- You may freely distribute the URL identifying the publication in the public portal 


\title{
Conformational stability study of a therapeutic peptide Plectasin using molecular dynamics simulations in combination with NMR
}

\author{
Sowmya Indrakumar ${ }^{1}$, Matja Zalar², Christin Pohl ${ }^{1,3}$, Allan Nørgaard ${ }^{3}$, Werner Streicher ${ }^{3}$, Pernille Harris ${ }^{1}$, \\ Alexander P. Golovanov², and Günther H.J. Peters ${ }^{1}$ \\ ${ }^{1}$ Technical University of Denmark, Department of Chemistry, 2800 Kgs. Lyngby, Denmark, \\ ${ }^{2}$ Manchester Institute of Biotechnology and School of Chemistry, The University of Manchester, Manchester M1 \\ 7DN, United Kingdom, ${ }^{3}$ Novozymes, Krogshoejvej 36, 2880 Bagsvaerd, Denmark
}

\begin{abstract}
Plectasin is a small, cysteine-rich peptide antibiotic, which belongs to the class of antibacterial peptides (AMPs) and has potential antimicrobial activity against various gram-positive bacteria. Groups of AMPs reported previously have shown that $\mathrm{pH}$ can modulate the antimicrobial mechanisms. In the current study, the effect of $\mathrm{pH}$ ranging from 4 to 9 , and $\mathrm{NaCl}$ concentrations of $70 \mathrm{mM}$, and $140 \mathrm{mM}$ on conformational stability of Plectasin, and three variants have been investigated. We show that at all studied physiochemical conditions, peptide secondary structures are intact during the 100ns simulations. However, flexibility in the loop close to the N-terminus increases with pH due to the change in electrostatics around the distinct anionic tetrapeptide stretch (residue numbers 9-12). Furthermore, Plectasin interactions with $\mathrm{NaCl}$ were measured by determining the preferential interaction coefficients, $\Gamma_{23}$. Generally, sodium ions have a high preference for the wild-type as residue 9ASP is mutated in all the three-peptide variants. Overall, the trend of $\Gamma_{23}$ with $\mathrm{pH}$ for the two salt conditions remain the same for all Plectasin variants and the common trend is that accumulation of sodium ions are observed for 10GLU and 12ASP. Insignificant changes in the overall peptide conformational stability is in agreement with the fact that Plectasin has three cystines. Thermodynamic integration molecular dynamics simulation supplemented with NMR chemical shift assays were employed to determine the degree of involvement of three different cystines to the overall structural integrity of the peptide. Both methods show the same order of cystines reduction and complete unfolding is observed only upon reduction of all cystines. Further, quantitative disulfide bond reduction assay shows a single transition of bond breakage with a significant lag phase implying breaking of the lowest energy cysteine bond is enough to drive the breaking of the remaining two-disulfide bonds.
\end{abstract}

\section{Introduction}

Peptide therapeutics are of interest for many pharmaceutical companies due to their potential to alleviate numerous severe diseases. They are highly specific with excellent efficacy, potency, and low toxicity as compared to small 
molecule drugs. ${ }^{1}$ Furthermore, peptide therapeutics are associated with lower production complexity, and hence their lower cost compared to protein therapeutics. ${ }^{2}$ However, their formulation poses various challenges, including self-association and aggregation, which can potentially lead to gel formation. ${ }^{3}$ Furthermore, peptides adopt multiple conformers and also exhibit a high degree of chemical instability. ${ }^{3}$ To overcome these issues, various stabilizing agents are used in an effort to stabilize peptides without losing structural integrity and biological activity. A common approach often includes a vast screening of different physiochemical conditions such as $\mathrm{pH}$, buffer systems, and addition of excipients. The challenge still remains to relate physiochemical conditions to structural properties of bio-molecules. Molecular dynamics (MD) simulation techniques have become an important method for bridging macroscopic solution properties with microscopic structural properties that allow for characterizing the physical basis of biomolecules' conformational stability and their biological functions. ${ }^{4}$ In the current study, the focus is on a therapeutic peptide called Plectasin, which belongs to the class of antibacterial peptides (AMPs). Amphipathic and cationic nature of AMPs allows them to interact with negatively charged bacterial surface components, leading to disruption of structural and functional integrity of membranes. ${ }^{5,6}$ Plectasin is the first defensin to be isolated from a fungus; the saprophytic ascomycete Pseudoplectania nigrella ${ }^{5}$ found on the ground of northern European pine forests. Plectasin has a tertiary structure that closely resembles those of defensins found in spiders, scorpions, and mussels ${ }^{5}$. Studies have shown that it is effective against several gram-positive bacteria, for example, Streptococcus pneumoniae, exerting bactericidal effects and curing pneumococcal peritonitis and pneumonia with low toxicity in mice ${ }^{5}$. Previous studies have shown that Plectasin binds directly to the pyrophosphate moiety of lipid II precursor constituting the cell well via hydrogen bonds with the amide hydrogens of 2PHE, 3GLY, 4CYS, and 37CYS. ${ }^{7}$ Plectasin is a 40 -amino acid long peptide that consists of an $\alpha-\beta$ motif comprising an $\alpha$-helix and two antiparallel $\beta$-strands (Figure 1). The structure is stabilized by three disulfide-bonds (4CYS-30CYS, 15CYS-37CYS, 19CYS-39CYS). Plectasin has a distinctive tetrapeptide stretch of anionic amino acids (residue numbers 9-12).

In the present study, MD simulation methods are explored to develop a computational approach that will provide the molecular understanding of the effect of $\mathrm{pH}$ and ionic strength (IS) on peptide structural dynamics. The effect of $\mathrm{pH}$ and IS on the wild-type Plectasin (hereafter referred to as PP40), and the three variants are investigated. These variants are i) ASN5SER ASP9ALA LYS26ARG, ii) ASP9SER GLN14LYS VAL36LEU and iii) ASP9ASN MET13LEU GLN14ARG which will hereafter be referred to PP41, PP42, and PP43, respectively. Additionally, the contribution of the three disulfide bonds to the conformational stability of wild-type Plectasin was investigated further using molecular dynamics simulation combined with thermodynamic integration methods in order to determine the free energy contribution from each of the cysteine bridges to the overall peptide stability. The computational study was complemented by a NMR titration study and a quantitative disulfide bond reduction assay. Good agreement is found between the computational and experimental results providing molecular insight into the order of cystines reduction and how cystines contribute to the overall structural integrity.

\section{Methods and Materials}




\section{In-silico methods}

\subsection{Constant pH MD simulations}

The Plectasin crystal structure of the wild-type (PDB ID: 3E7 ${ }^{8}$ ) was used as a template to introduce point mutations to construct models of the three Plectasin variants. In all mutants, certain residue was mutated to either a positive charged polar amino acid or a hydrophobic amino acid in an attempt to improve its ability to bind to the negatively charged gram-positive microorganisms. ${ }^{2,9}$ Additionally, PP43 has 13MET mutated to LEU making it less prone to oxidation. ${ }^{10,11}$ Figure 1A shows the sequence alignment of Plectasin variants along with disulfide bonds connectivity and secondary structure assignment.

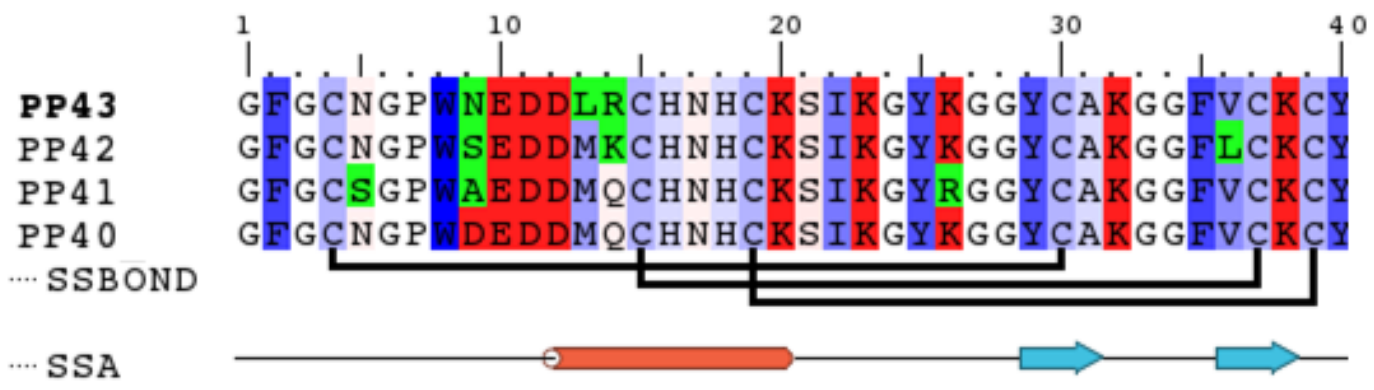

Figure 1: A. Sequence alignment of wild-type Plectasin and the three mutants. Residues are colored based on hydrophilicity, and mutations are marked in green. The disulfide bridges (SSBOND) are shown as black lines, and secondary structure assignment (SSA) shows position of secondary structural elements. Alpha helix is shown as red cylinder, beta strands as blue arrows and loop regions as black lines.

Peptide structures were minimized with the PrepWiz tool from Schrödinger release 2016-3 (Schrödinger, LLC, New York, NY, USA). ${ }^{12}$ The protonation state of the titratable amino acids at different $\mathrm{pH}$ and IS values were calculated using the $\mathrm{H++}$ server (http://biophysics.cs.vt.edu/H++ ). ${ }^{13}$ The server settings were adjusted to an internal dielectric constant of 10 which is appropriate for residues close to the surface and external dielectric constant of 80 for water, respectively. ${ }^{13}$ The $\mathrm{pH}$ range was from 4 to $9(\Delta \mathrm{pH}=1)$ with salinity adjusted to $0 \mathrm{mM}, 70 \mathrm{mM}$, and $140 \mathrm{mM}$. The topology and coordinate files generated as output in Amber format were used to generate the PDB structure file using ambpdb tool implemented in Amber. ${ }^{14}$ The PDB file was used as the starting structure for molecular dynamics simulations in Amber. All-atom classical constant $\mathrm{pH}$ molecular dynamics (MD) simulations ${ }^{15}$ in explicit solvent were carried out with the Amber 16 program $^{16}$ employing the amber force field ff99SB ${ }^{17}$ for proteins. Water molecules were represented using the TIP3P ${ }^{18}$ water model. All bonds to hydrogen atoms were constrained using the SHAKE algorithm. ${ }^{19}$ For each of the peptide systems, 18 different physicochemical conditions were tested. Each system was neutralized using either sodium or chloride ions depending on the overall charge state and solvated in a truncated octahedron water box with a $15 \AA$ padding in all directions. IS was adjusted to $70 \mathrm{mM}$ and $140 \mathrm{mM}$ by additions of equal number of sodium and chloride ions. To adjust the IS to $70 \mathrm{mM}$ and 140mM, 9 and 18 ions were 
added to the system, respectively. In total, the solvated system contained approximately 7100 atoms. The particle mesh Ewald method ${ }^{20}$ was employed to determine the non-bonded electrostatics energies with a real-space cutoff of $8 \AA$. The system was minimized for 5000 steps. The first 1500 steps were performed using the steepest descent method followed by the conjugate gradient method for the remaining steps. Subsequently, the system was heated linearly from $10 \mathrm{~K}$ to $300 \mathrm{~K}$ within $1 \mathrm{~ns}$ using Langevin thermostat ${ }^{21}$ with a collision frequency of $5 \mathrm{ps}^{-1}$. Berendsen barostat $^{22}$ was used to control pressure dynamics. The systems were then equilibrated for 4 ns at constant temperature (300 K) and pressure (1 bar). Coordinates were saved every 5 ps for further analyses. Finally, constant $\mathrm{pH}$ simulations were performed for 100 ns and coordinates were saved every $10 \mathrm{ps}$. The protonation state of titratable residues were fixed during the simulations since the initial peptide variants were modeled at the desired $\mathrm{pH}$ and ionic strength. Each system at a particular physiochemical condition was simulated in duplicates for $100 \mathrm{~ns}$ starting from a random seed number to estimate the statistical uncertainty of the results.

Analyses were performed with CPPTRAJ ${ }^{23}$ in Amber16, and VMD 1.9.324. Clustering method was employed as a mean of grouping similar objects into one cluster. In the perspective of MD simulations, conformations generated over time were grouped into distinct clusters depending on the conformational similarity. In this study, the agglomerative hierarchical clustering approach has been incorporated that start with treating each data point as a singleton cluster and then successively merge nearest clusters until all clusters have been grouped into the desired number of clusters. ${ }^{25,26}$ The cluster proximity is defined based on average-linkage, where the inter-cluster distance is defined as the average root mean square deviation (RMSD) distance measured between every data point from one cluster to all points in the other cluster. Additionally, principal component analysis (PCA) ${ }^{27-29}$ was employed to explore the dynamics of the peptides. PCA was extended further to obtain free energy landscapes as a function of the first few principal components, in our case, PC1 and PC2. Two-dimensional histogram analysis is performed on the PCs data points and free energy for each bin is estimated using

$$
G_{\mathrm{i}}=k_{\mathrm{B}} T \ln (P(\mathrm{PC} 1, \mathrm{PC} 2)
$$

Where $T$ is the temperature in Kelvin, $k_{\mathrm{B}}$ is the Boltzmann constant, and $P(\mathrm{PC} 1, \mathrm{PC} 2)$ is the probability distribution of the sampled structures along the first and second eigenvectors.

\subsubsection{Preferential Interaction Coefficient $\left(\Gamma_{23}\right)$}

The preferential interaction coefficient was used to estimate the binding capacity of ions to surface of Plectasin variants. The measure of preferential interactions between co-solvents and peptide have been used to deduce the effects of co-solvents on the molecular systems ${ }^{30}$. In this study, the statistical mechanics definition of $\Gamma_{23}$ is used to describe peptide co-solvent interactions ${ }^{31}$.

$$
\Gamma_{23}=\left\langle n_{3}^{I I}-n_{1}^{I I}\left(\frac{n_{3}^{I}}{n_{1}^{1}}\right)\right\rangle
$$


where $n$ denotes the number of specific molecules. Subscript 1, 2 and 3 stand for water, peptide, and co-solvent, respectively. Superscript I represents a bulk region, which is outside of the vicinity of the peptide. Superscript II represents a local region located near the peptide, and angled brackets $\langle>$ denote an ensemble average. Favorable peptide co-solvent interactions result in a positive $\Gamma_{23}$ implying the concentration of the co-solvent is higher in the local domain as compared to the bulk domain and vice-versa when $\Gamma_{23}$ is negative. A cutoff of $8 \AA$ was used to differentiate local from the bulk domain based on $\mathrm{g}(r)$ calculation for $\mathrm{Cl} / \mathrm{Na}^{+}$with respect to charged and polar parts of the peptide (data not shown). Trajectories of the production runs were divided into smaller parts each containing 50 frames ( $5 \mathrm{~ns}$ ), standard error of the means of these several parts is the estimated error in $\Gamma_{23}$.

\subsection{Disulfide bonds contribution to the conformational stability}

Classical all-atom MD simulations were performed on wild-type Plectasin where each of the cystine bonds was individually broken or all cystines at the same time. The same MD setup was used as for constant $\mathrm{pH}$ MD simulations described above. Simulation conditions were chosen to be $\mathrm{pH} 4$ and $0 \mathrm{mM}$ IS corresponding to the experimental conditions used in NMR. This study was complemented with TI-MD simulations to estimate the relative free energy cost of breaking the disulfide bonds. The free energy change for breaking the disulfide bonds is estimated by transforming from cystines (State A) to free cysteines (State B), by coupling the two states via a parameter $\lambda$. The free energy difference in going from State A $(\lambda=0)$ to State $B(\lambda=1)$ is computed as follows:

$$
\Delta G(A \rightarrow B)=\int_{0}^{1}\left\langle\frac{\partial V(\lambda)}{\partial \lambda}\right\rangle \partial \lambda
$$

The change in free energy is computed from the integral of the ensemble averaged derivates of the potential energy function $V(\lambda)$ with respect to $\lambda$. Five independent simulations were performed to study the energetic cost for breaking the disulfide bonds. Different scenarios were tested, and simulations were carried out where each of the disulfide bonds was broken independently, and where all three bonds were broken. The parameter $\lambda$ was varied successively over 11 values $(0.005 / 0.05,0.1,0.2,0.3,0.4,0.5,0.6,0.7,0.8,0.9,0.95 / 0.995)$ where each $\lambda$ was simulated for $20 \mathrm{~ns}$. It is crucial to chose the end $\lambda$ points close to 0 and 1 carefully to allow smooth extrapolation. The system with $\lambda=0.5$ was minimised, heated linearly to $300 \mathrm{~K}$ over a period of $400 \mathrm{ps}$. This structure was subsequently used for the equilibration and production runs for all $\lambda s$. The bond dissipating the least energy during the TI-MD breaks first. Consequently, to understand the sequential breaking, bond with least energy penalty is kept broken through out the next simulations, each of the remaining two cysteine bridges were transformed linearly as described above in independent simulations. Surface accesibile surface areas (SASAs) were calculated for each representative structure of the simulated system using GetArea ${ }^{32}$ with a probe radius of $2.8 \AA$ corresponding to the effective radius of the reducing agent used in the experiments.

\section{Experimental methods}

\subsection{Quantitative assay to measure free cysteines}


Plectasin samples were provided by Novozymes A/S. The assay was developed after the protocol of Thanhauser et al. ${ }^{33}$ Plectasin was dialyzed into $10 \mathrm{mM}$ Histidine buffer $\mathrm{pH} 4.5$ as peptide is stable only at low $\mathrm{pH}$, at $4^{\circ} \mathrm{C}$ using slide-a-lyzer 2000 MWCO dialysis cassettes (Thermo Scientific) for 20 hours with two times buffer exchange after 2 and 4 hours, respectively. The final concentration of the dialyzed sample was determined by measuring UV absorbance at $280 \mathrm{~nm}$ with a Nanodrop ${ }^{\mathrm{TM}}$ spectrophotometer (Thermo Scientific), using an extinction coefficient of 2.24 .

A final peptide concentration of $200 \mu \mathrm{M}$ was prepared by diluting the dialyzed peptide using buffer from the last dialysis reservoir. Half of the prepared $200 \mu \mathrm{M}$ peptide sample was denatured using 3M GnHCl. A concentration series of $25-200 \mu \mathrm{M}$ with increments of $25 \mu \mathrm{M}$ of the native and the denatured sample were prepared in duplicates in a microtiter plate. As a control, a concentration series with the same concentrations of oxidized glutathione (Sigma-Aldrich) was prepared. The baseline was determined using $\mathrm{H}_{2} \mathrm{O}$ instead of protein stock solution. $250 \mu \mathrm{l}$ of the NTSB (disodium 2-nitro-5-thiosulfobenzoate) assay solution (200mM TRIS, 100mM Na $2 \mathrm{SO}_{3}$, 3mM EDTA pH 9.5 plus $1 \%$ NTSB stock solution (100 mg DTNB (Sigma-Aldrich) dissolved in $10 \mathrm{ml} 1 \mathrm{M} \mathrm{Na}_{2} \mathrm{SO}_{3} \mathrm{pH} 7.5$; oxygen bubbled through at $38^{\circ} \mathrm{C}$ )) was added to the peptide samples. The reaction was monitored by recording the absorption at $412 \mathrm{~nm}$.

\subsection{NMR experiments}

The peptide sample was extensively dialyzed into $20 \mathrm{mM}$ citrate buffer, $\mathrm{pH} 4.0$, at $4{ }^{\circ} \mathrm{C}$ using slide-a-lyzer 2000 MWCO dialysis cassettes (Thermo Scientific) until the original formulation components were no longer detectable in the ${ }^{1} \mathrm{H}$ NMR spectrum. After dialysis, $5 \%{ }^{2} \mathrm{H}_{2} \mathrm{O}$ was added to the sample. The final concentration of Plectasin was $2 \mathrm{mM}$ as determined by measuring UV absorbance at $280 \mathrm{~nm}(\varepsilon=2.24)$ with a Nanodrop ${ }^{\mathrm{TM}}$ spectrophotometer (Thermo Scientific).

All NMR experiments were acquired on Bruker $800 \mathrm{MHz}$ Avance III spectrometer equipped with $5 \mathrm{~mm}$ TCI cryoprobe and temperature control unit at $25{ }^{\circ} \mathrm{C}$. The $\mathrm{C}_{\alpha}$ assignments of Plectasin were transferred from BMRB entry 16739 to the $2 \mathrm{D}{ }^{1} \mathrm{H}^{-13} \mathrm{C}$ HSQC, then the side chain assignment of each amino acid was achieved using a 2D ${ }^{1} \mathrm{H}-{ }^{13} \mathrm{C}$ heteronuclear single quantum correlation - total correlated spectroscopy (HSQC-TOCSY) spectra of Plectasin. 2D data matrices with $8 \mathrm{k}$ x $1 \mathrm{k}$ complex points were collected for both experiments with a spectral width of 16.0 and $165 \mathrm{ppm}$ for ${ }^{1} \mathrm{H}$ and ${ }^{13} \mathrm{C}$ dimensions, respectively. All signals, except $\mathrm{H}_{\alpha}$ atoms of 4CYS, 8TRP, 10GLU, 11ASP, 15CYS, 25TYR, 29TYR, and 31ALA, were ,unambiguously assigned. Gradual reduction of disulfide bonds was achieved by stepwise addition of tris(2-carboxyethyl)phosphine (TCEP) to the plectacin sample from a $1 \mathrm{M}$ stock solution, to minimize dilution effects. TCEP was used since the strong reducing agent is still effective at $\mathrm{pH}$ 4.0.

At each point of the titration, one dimensional (1D) proton $\left({ }^{1} \mathrm{H}\right)$ experiment with excitation sculpting water suppression and two dimensional (2D) ${ }^{1} \mathrm{H}-{ }^{13} \mathrm{C}$ - HSQC experiment with sensitivity enhancement, gradient coherence selection, and multiplicity editing was acquired. For all 1D ${ }^{1} \mathrm{H}$ experiments, 32k complex points were collected with 
spectral window $16.0 \mathrm{ppm}$, while 2D data matrix of $4 \mathrm{k}$ x 512 complex points with spectral windows $16.0 \mathrm{ppm}$ for ${ }^{1} \mathrm{H}$ and $165 \mathrm{ppm}$ for ${ }^{13} \mathrm{C}$ dimension were acquired. Intensity data as a function of concentration of TCEP for all the Plectasin assigned peaks were then fitted to the logistic function:

$$
I(c)=A_{1}+\frac{A_{2}-A_{1}}{\left[1+\left(\frac{c}{c_{1 / 2}}\right)^{p}\right]}
$$

where $\mathrm{c}$ is the concentration of TCEP, $\mathrm{c}_{1 / 2}$ is the midpoint of the sigmoidal curve, $\mathrm{p}$ is the steepness of the curve, and $\mathrm{A}_{1}$ and $\mathrm{A}_{2}$ are lower and upper asymptotes, respectively. $c_{1 / 2}$ in this case indicates the concentration of TCEP needed for $50 \%$ transition of each amino acid into the unfolded state, also known as $\mathrm{EC}_{50}$.

\section{Results and Discussion}

\subsection{MD simulation}

To map the conformational properties of Plectasin, MD simulations of Plectasin were performed at different $\mathrm{pH}$ and ionic strengths. This amounted to eighteen different physiochemical conditions, where each system was simulated in duplicates for 100 ns to estimate for statistical uncertainty of the results. The overall stablity of Plectasin was assessed by calculating the root mean square deviation (RMSD). For all simulations at the different physicochemical conditions, the RMSDs converged after $\sim 25$ ns reaching plateaus in the range $0.5-3 \AA$. There was no correlation between the overall RMSDs and $\mathrm{pH}$ and ionic strengths.

\section{Effect of $\mathrm{pH}$}

The effect of $\mathrm{pH}$ on the conformational stability was studied using the constant-pH MD protocol in Amber version 16. For all the Plectasin variants, at $\mathrm{pH} 4$ peptide prefers to be in its "native crystal structure conformation". On the other hand, at higher $\mathrm{pH}$, peptides were more flexible. Figure 2 represents the free energy landscape (FEL) for the peptides at pH 9. The surface has multiple well-defined basins indicating different conformations that the peptides explore during the course of the simulations. However, the energy barriers between the basins are small, and the peptide can leap from one conformation to the other. The motion of the flexible regions are shown as mode vectors in Figure S1. A major contribution to flexibility comes from the loop region close to the N-terminus, which stays almost intact at low pH (data not shown). The loop dynamics could be due to the change in the protonation state of titratable residues. In the working $\mathrm{pH}$ range, a change in the protonation state of 10GLU, 16HIS, and 18HIS is observed. While both histidines are charged at $\mathrm{pH} 4,16 \mathrm{HIS}$ deprotonates already at $\mathrm{pH}$, and 18HIS deprotonates at $\mathrm{pH}$ 9. Whereas, $10 \mathrm{GLU}$ is deprotonated at $\mathrm{pH} 4$, and is protonated already at $\mathrm{pH} 5$. This leads to a noticeable change in the surface electrostatics (Figure 3). At pH 4, all variants, except PP42, have conformations present in one welldefined basin, while for the PP42 variant, the FEL has two basins (Figure S2), but the conformations are very similar with low RMSD of less than $1 \AA$. 

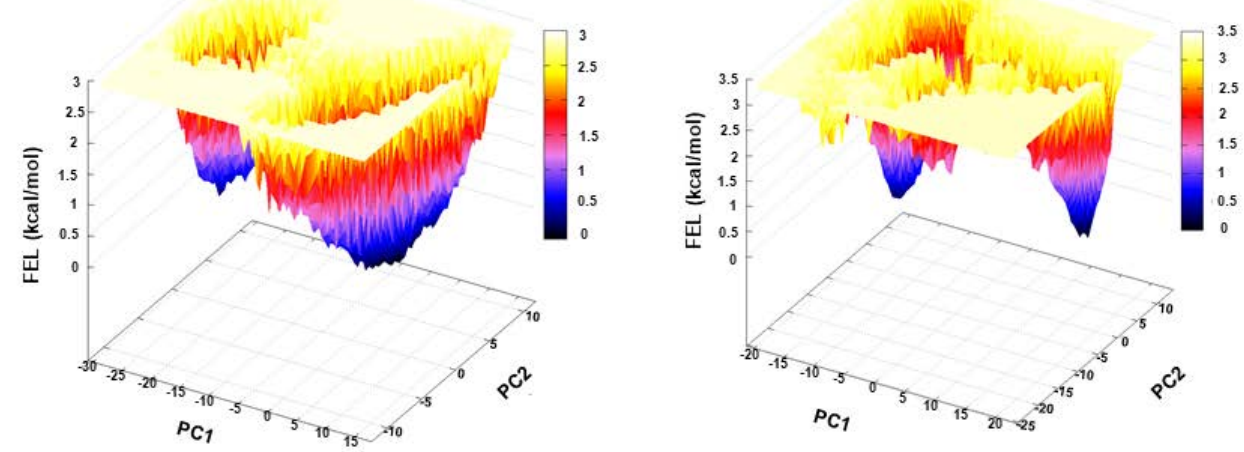

PP42

PP43
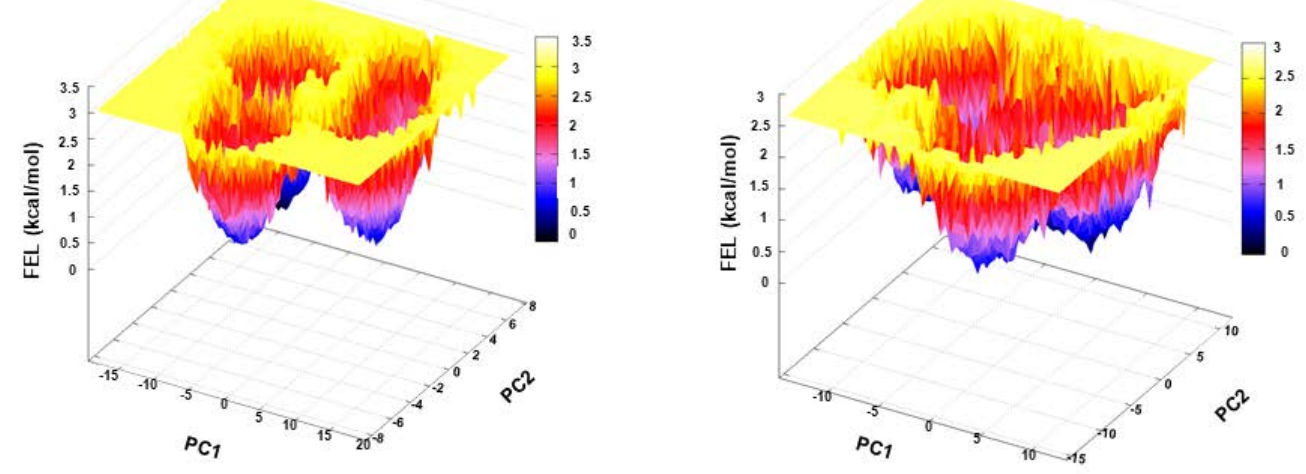

Figure 2. Three-dimensional FEL displayed as a function of the first two principal components for all the four Plectasin variants at $\mathrm{pH} 9$. 


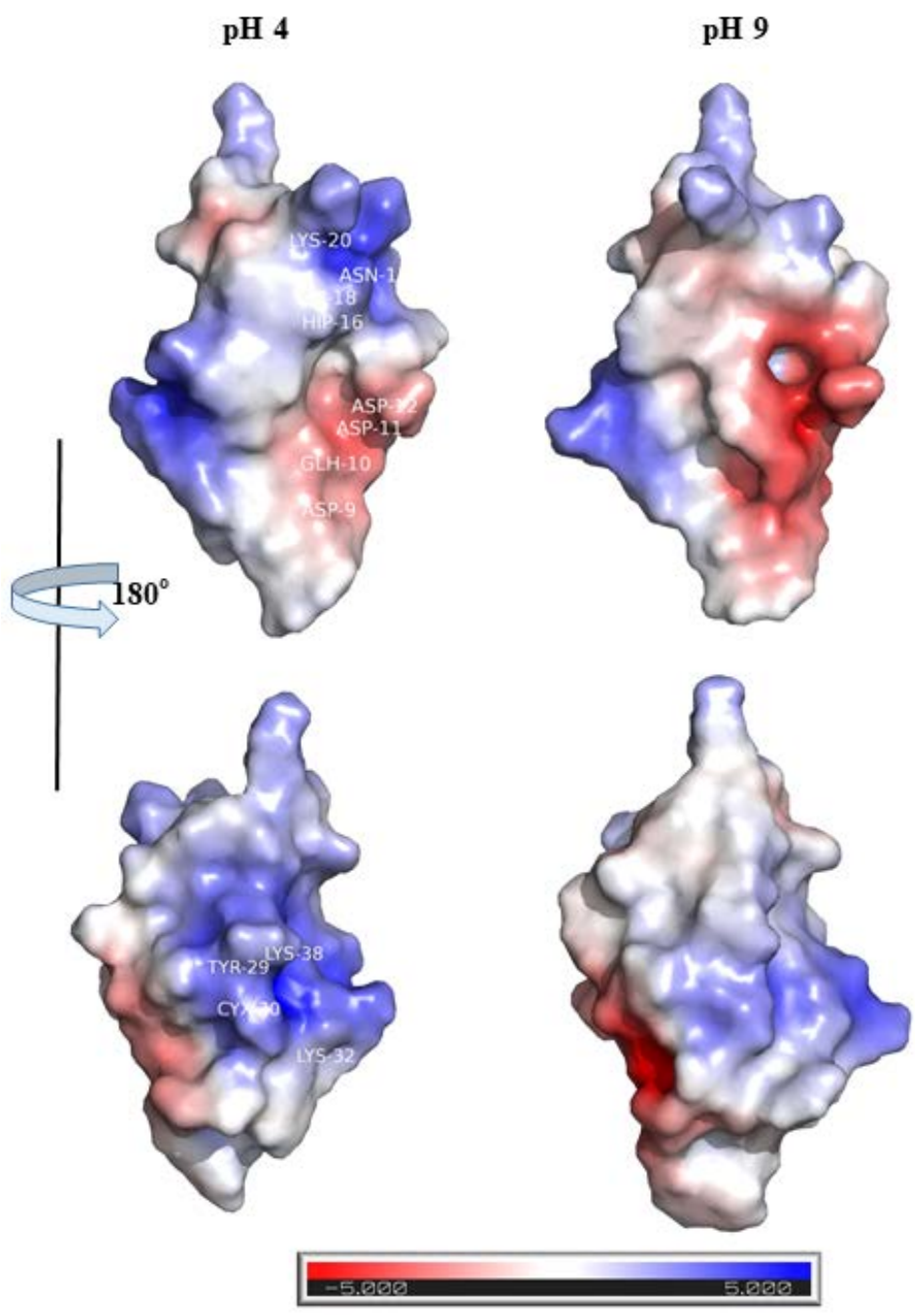

Figure 3. Electrostatic surface representation of structures at $\mathrm{pH} 4$ (left) and $\mathrm{pH} 9$ (right) respectively for PP40. At $\mathrm{pH}$ 9, electrostatic surface coloration shows accumulation of electronegative charges and reduction of positive charges in the tetrapeptide segment (ASP-GLU-ASP-ASP) and histidine region, respectively. The same change in electrostatics is observed for PP41, PP42 and PP43 (data not shown). PDB2PQR ${ }^{34}$ server was used to generate electrostatic surface plots.

\section{Effect of $\mathrm{NaCl}$}

The preferential interaction coefficient $\left(\Gamma_{23}\right)$ was used to estimate the binding capacity of ions to surface of Plectasin variants. The measure of preferential interactions between the co-solvents and peptide have been used to deduce the effects of co-solvents on the molecular systems. ${ }^{30}$ Plectasin variants have different preferential interaction with sodium and chloride ions. The trend of $\Gamma_{23}$ with $\mathrm{pH}$ remains the same for the two salt concentrations, i.e., $70 \mathrm{mM}$ and $140 \mathrm{mM} \mathrm{NaCl}$ (Figure 4). Generally, differences in the $\Gamma_{23}$ are arising from the change in the electrostatics of the ASP-GLU-ASP-ASP segment. For all variants, the interaction scores are high for the GLU-ASP-ASP compared to the other residues, which implies preferential interactions of sodium ions with this region. Moreover, ASP-GLUASP-ASP stretch and other loop segments are more exposed compared to the residues that constitute $\alpha \beta$ secondary structures. Interaction of lysines with chloride ions are also observed, but interactions are lower as compared to sodium ions which reflects that sodium ions have a higher attraction for negatively charged residues than chloride 
ions for positively charged residues. In other words, chloride ions do not prefer to be on the peptide surface, which is also observed for other proteins. ${ }^{35}$ In the following, the differences in $\Gamma_{23}$ for each of the peptide variants is discussed.
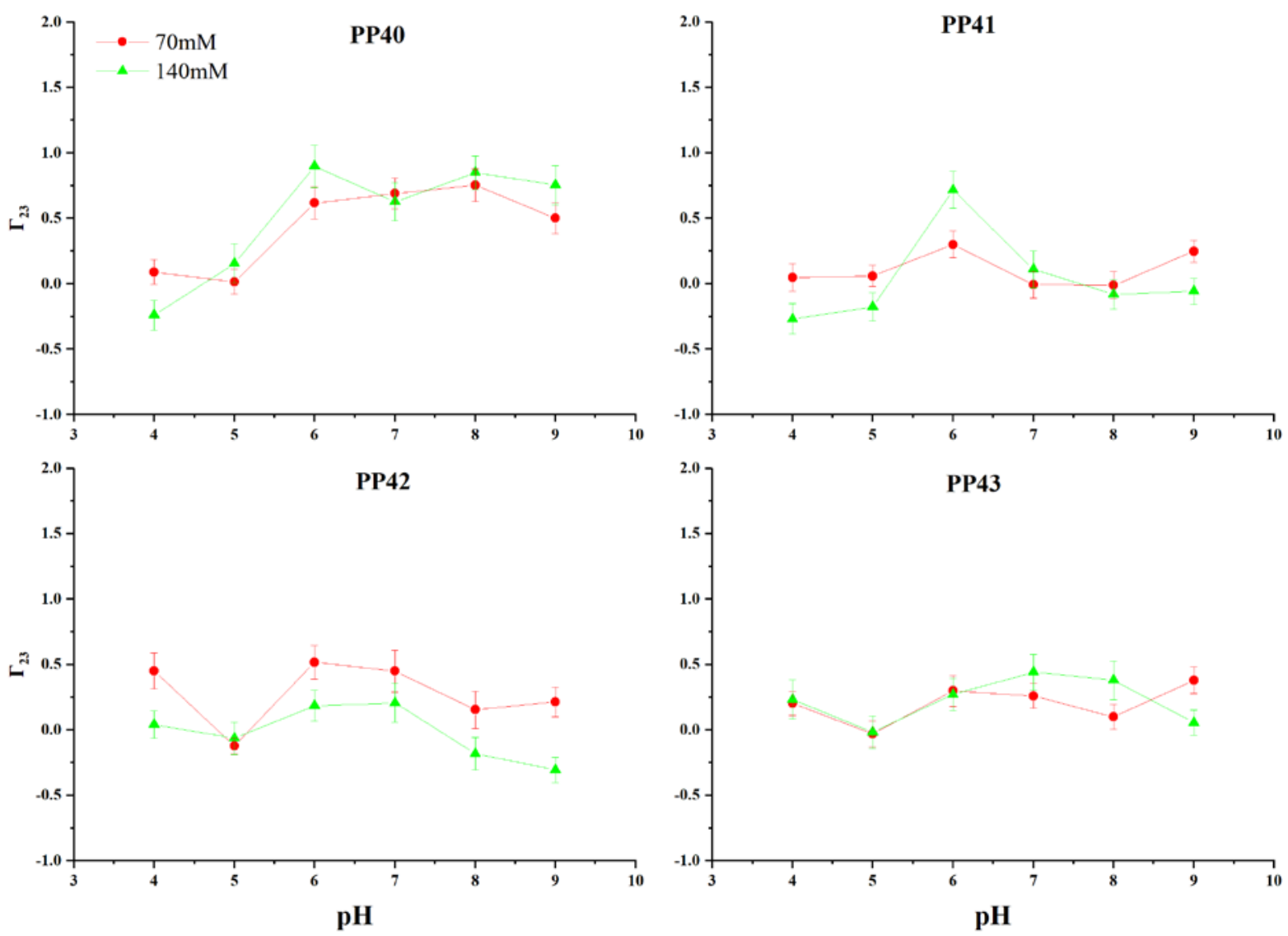

Figure 4. $\Gamma_{23}$ of all the Plectasin variants are displayed as a function of $\mathrm{pH}$ for the two salt conditions, $70 \mathrm{mM}$ (red) and $140 \mathrm{mM}$ (green) $\mathrm{NaCl}$.

In the case of PP40, a noticeable increase in $\Gamma_{23}$ is observed between $\mathrm{pH} 5$ and 6 , which is less pronounced for the other Plectasin variants. To further investigate the contribution of residues to $\Gamma_{23}$, an interaction score $\left(I_{s c o r e}\right)$ was defined as follows:

$$
P\left(I_{\text {score }}\right)=n /(N * a)
$$

where $n$ denotes the number of times either sodium or chloride was in contact with a certain residue over simulation time, $N$ is for a total number of frames, $a$ is a normalization factor which ensures that the interaction score is converted into probability. Note that $\Gamma_{23}$ defines the overall accumulation of salt to the protein surface, whereas $P\left(I_{\text {score }}\right)$ defines interactions per residue. Uncertainties seen in $P\left(I_{\text {score }}\right)$ are due to conformational flexibility of the peptides, which is dependent on the physiochemical conditions and the dynamics of ion's absorption and desorption. 
$P\left(I_{\text {score }}\right)$ are depicted in Figure 5, and indicate that residues 9ASP, 10GLU, 11ASP, 12ASP, 23LYS, 30CYS, 32LYS, 38LYS have increased preference for co-solutes at higher $\mathrm{pH}$ as compared to $\mathrm{pH} 4$. In particular, the ASPGLU-ASP-ASP (9-12) region shows relatively high scores. This is expected, since this region forms a negatively charged patch on the peptide surface attracting positively charged sodium ions. The electrostatic properties of this region can also explain the change in $\Gamma_{23}$ between $\mathrm{pH} 5$ and 6 . Due to the cluster of negatively charged residues, the $p K_{\mathrm{a}}$ of $10 \mathrm{GLU}$ is shifted upwards; i.e. that $10 \mathrm{GLU}$ becomes deprotonated (i.e. charged) when the $\mathrm{pH}$ is changed from 5 to 6. The change in $\Gamma_{23}$ is less pronounced for PP41, PP42 and PP43 than PP40 since 9ASP is mutated to either a polar or hydrophobic amino acid leading to a decrease in $P\left(I_{\text {score }}\right)$ at this position.

Considering the statistical uncertainties, $\Gamma_{23}$ for PP40, PP41 and PP43 are comparable at $70 \mathrm{mM}$ and $140 \mathrm{mM} \mathrm{NaCl}$ implying that the peptide surface is not saturated. In the case of PP42, $\Gamma_{23}$ values are lower for $140 \mathrm{mM}$ in comparison to $70 \mathrm{mM}$, implying that salt prefers to be in the bulk. Looking at eq 2, $n_{1}^{I I}$ (salt in the bulk) increases leading to lowering of $\Gamma_{23}$. The common trend seen for all variants is that 10GLU and 12ASP are the common residues of interaction with sodium ions.
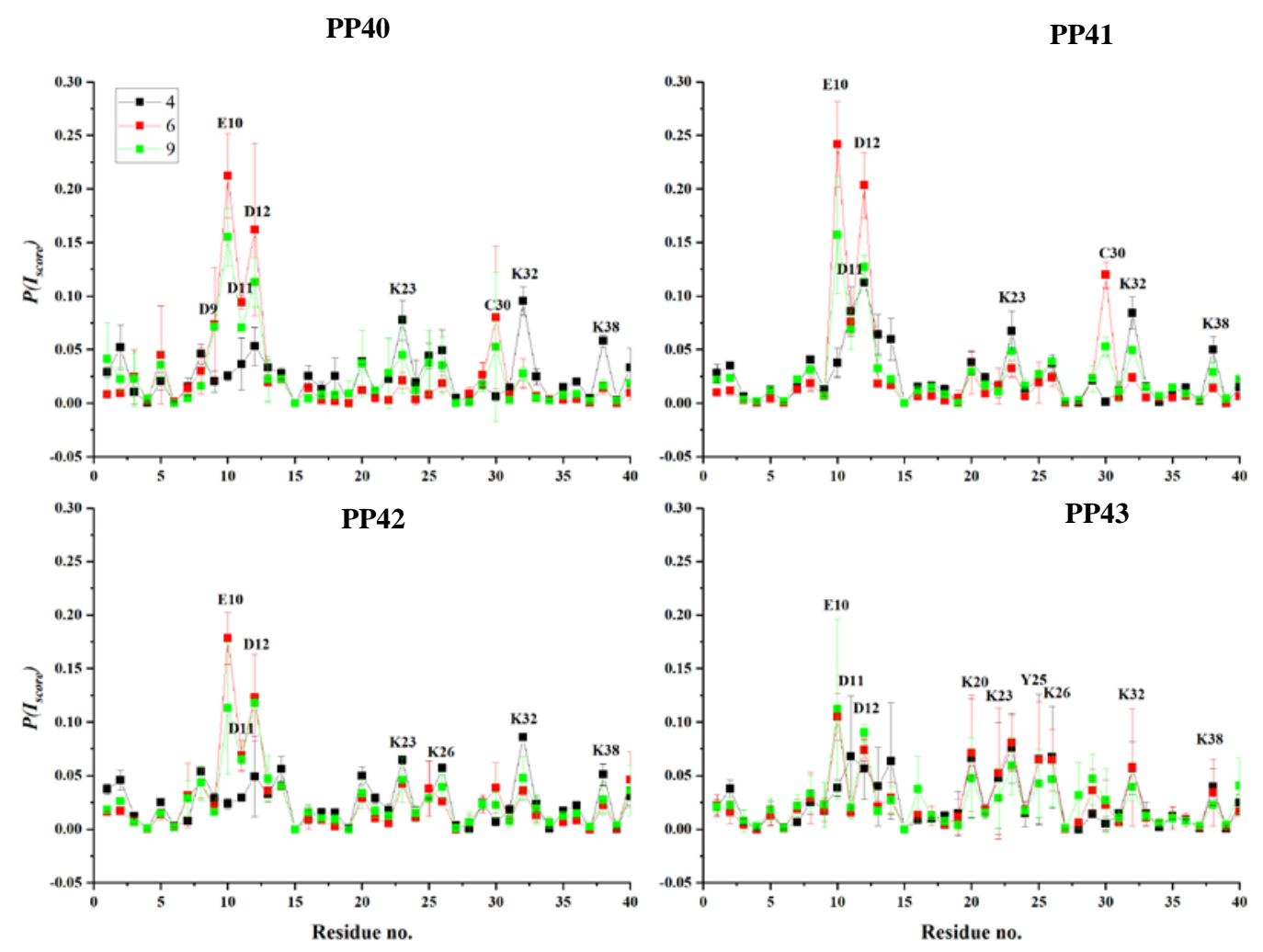

Figure 5: Interaction score calculated per residue for $140 \mathrm{mM} \mathrm{NaCl}$ salt concentrations to have sufficient crowding for all the Plectasin variants for $\mathrm{pH} 4$ (black), pH 6 (red) and pH 9 (green). The interaction score is calculated as an average over two replicates. 


\subsection{Role of cystines in conformational stability of Plectasin}

MD simulations at different physiochemical conditions showed that the peptides undergo moderate flexibility in the loop region, which is in connection with the fact that Plectasin has three cystines. In this section, the contribution from each of the disulfide bridges on the overall conformational stability was explored using classical all-atom MD and thermodynamic integration molecular dynamics (TI-MD) simulations in Amber16. ${ }^{36,37}$ This work is complemented with NMR titration experiments and a quantitative assay, which measures change in absorbance upon formation of free cysteines in solution.

From the TI-MD simulations $\Delta G$ was extracted for each of the simulated systems and are summarized in table I. The least $\Delta G$ of $0.04 \mathrm{kcal} / \mathrm{mol}$ is obtained for the system, which is transformed linearly from three pairs of cystines to the system where 15CYS-37CYS is broken. SASA of 15CYS-37CYS is $16.5 \AA^{2}$, which is higher compared to 4CYS-30CYS and 19CYS-39CYS having SASA of $12.1 \AA^{2}$ and $8.0 \AA^{2}$, respectively. Further, breakage of 15CYS37CYS bond does not lead to significant structural changes, which is also reflected in low RMSD (Figure 6A). Furthermore, the change in loop entropy is minimal upon reduction of 15CYS-37CYS as the other two cystines are present keeping structure rather intact. Breaking of 15CYS-37CYS leads to insignificant change in exposure of the apolar surface area, which is $2245 \AA^{2}$ as compared to the $2210 \AA^{2}$ for the WT. Upon reduction of 15CYS-37CYS, the polar SASA increases by $\sim 6 \%$. The relatively low reduction in the apolar SASA, indicates that the reduction of the cystine is enthalpic driven rather than entropic driven. ${ }^{38}$

Considering the aforementioned information, keeping 15CYS-37CYS broken throughout the simulation, linear transformation of the systems where the other two cystines are intact to a system where either of the two cystines are broken shows that the second preferential bond to break is 4CYS-30CYS. Moreover, comparing the accessible

Table I. $\Delta G$ derived from TI-MD simulations for the different scenarios of breaking disulfide bonds.

\begin{tabular}{ll}
\hline System & $\Delta \boldsymbol{G}(\mathbf{k c a l} / \mathbf{m o l})$ \\
\hline All 3 bonds broken & 3.56 \\
4CYS-30CYSbroken & 2.16 \\
15CYS-37CYS broken & 0.04 \\
19CYS-39CYSbroken & 0.35 \\
\hline \multicolumn{2}{l}{ 15CYS-37CYS broken throughout TI-MD } \\
\hline $\begin{array}{l}\text { 4CYS-30CYS broken } \\
\text { 19CYS-39CYS broken }\end{array}$ & 4.65 \\
\hline
\end{tabular}
surface area of the structure with 15CYS-37CYS broken shows that, 4CYS-30CYS has a SASA of $47.1 \AA^{2}$ compared to $4.9 \AA^{2}$ for 19CYS-39CYS. Upon breaking of the second cystine, there is an increase in exposure of polar surface area to $2598 \AA^{2}$. Therefore, the order of sequential breaking is 15CYS-37CYS, 4CYS-30CYS, and 19CYS-39CYS. Figure S3 shows the curves of $\partial V / \partial \lambda$ as a function of $\lambda$ step for the systems simulated using TI-MD method. Independently, classical all-atom simulations were performed on systems having each of the cystines broken. Simulations showed that breaking of all three cystines initiates peptide unfolding, and breaking of each of the three bonds independently does not lead to loss of secondary structural components during the simulation (Figure 6B). The relatively high RMSD for the 
system where 4CYS-30CYS is broken origins from the free end at the N-terminus, however, the secondary structures stay intact (Figure 6B).

A.
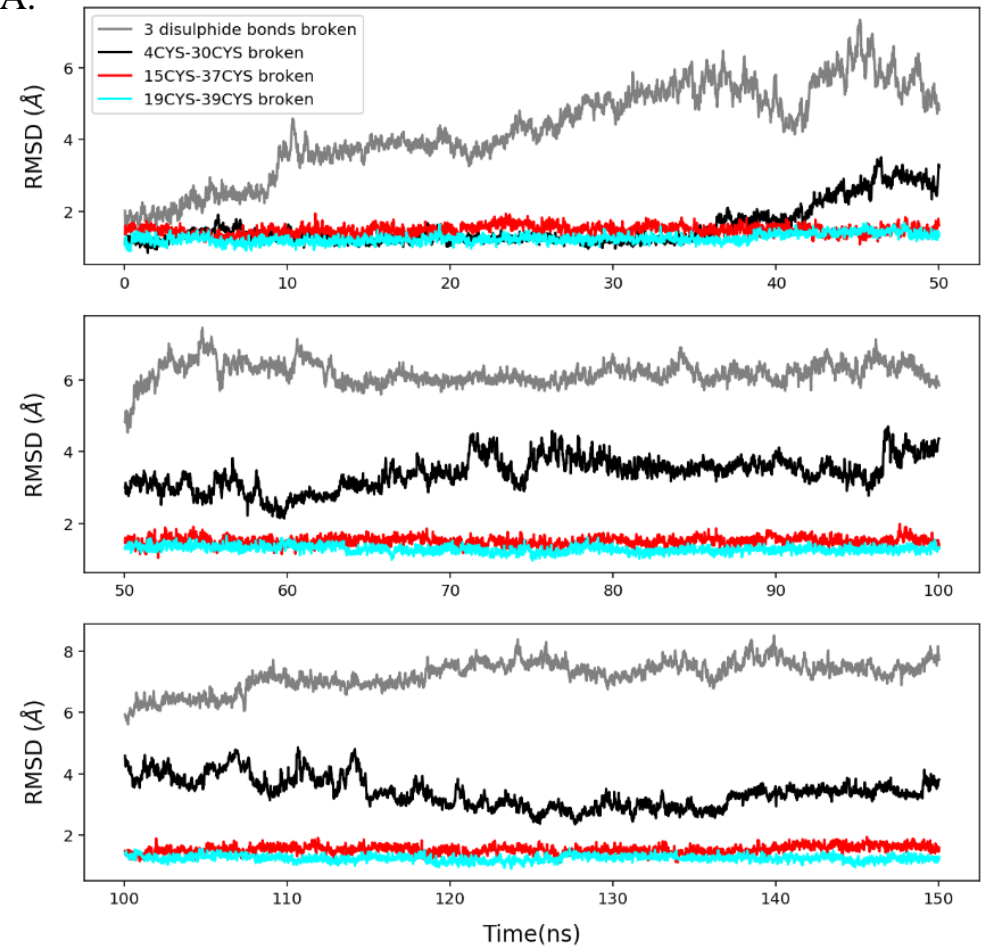

B.

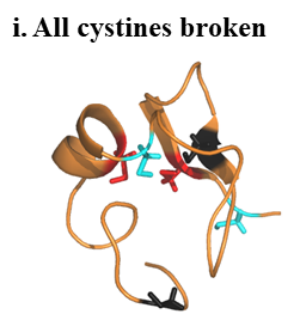

ii. 4 CYS-30CYS broken

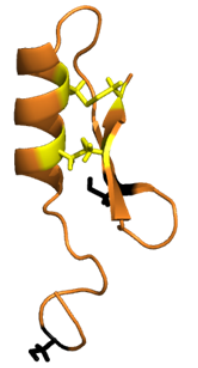

iii. 15CYS-37CYS broken iv. 19CYS-39CYS broken
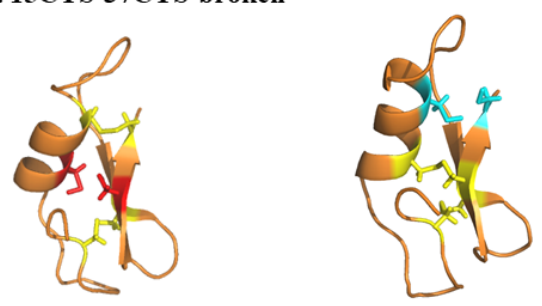

Figure 6: A. Plot showing average RMSD based on two replicate runs of the simulated systems, i.e., all cysteine bonds are broken (gray), 4CYS-30CYS broken (black), 15CYS-37CYS broken (red), and 19CYS-39CYS broken (cyan), respectively. B. Shows final structures after 150ns of simulation for each of the simulated systems. Broken disulfide bonds are marked in black (4CYS-30CYS), red (15CYS-37CYS), and cyan (19CYS-39CYS).

\subsection{Monitoring disulfide reduction}

Initially, the disulfide breakage reaction was performed at $25^{\circ} \mathrm{C}$. However, the kinetics of reaction were too slow to be observed within the experimental time frame. Therefore, the temperature was raised to $40^{\circ} \mathrm{C}$ (comparison of the two temperatures measurements is discussed briefly in supporting information; Figure S4). Noticeably, Figure 7 shows an initial lag phase for the Plectasin that reflects a delay in the breakage of one or more disulfide bonds. The lag phase is reduced in the denatured Plectasin as the disulfide bridges are more exposed than in the native Plectasin. This result reveals that the lag phase could be due to the steric protection of the disulfide bridges, which disappears with the breakage of the first disulfide bond, eventually leading to the breakage of the remaining disulfide bridges. Another reason could be the change in hydration entropy of loops upon the breakage of the first disulfide bond leading to a destabilization of the remaining disulfide bonds. ${ }^{38-40}$ Upon breakage of the first cystine which 15CYS37CYS, SASA of the other two cystines, i.e. $47.1 \AA^{2}$ for 4CYS-30CYS compared to $4.9 \AA^{2}$ for 19CYS-39CYS, increases making it more accessible to NTSB, which in turn increases the apolar surface area leading to change in 
hydration entropy, and thus, reduction of other two cystines. ${ }^{38}$ Moreover, smooth transition from native bonded state to broken cysteines indicates a bond energy for the breaking of three cystines are close.

Oxidised glutathione, that has one disulfide bridge, was used as the reference in order to correlate concentration with the number of disulfide bridges broken, and the plateau at $30 \mu \mathrm{M}$ of NTB corresponds to the breakage of one disulfide bridge (Figure 7). From this, the number of disulfide bridges in Plectasin is calculated as 2.6. In this assay, a value of approx. 2.6 of disulfide bridges for both the native and denatured form of Plectasin is observed. This is close to the expected value of 3 disulfide bridges. The difference could arise from experimental uncertainties with respect to protein and NTSB concentrations.

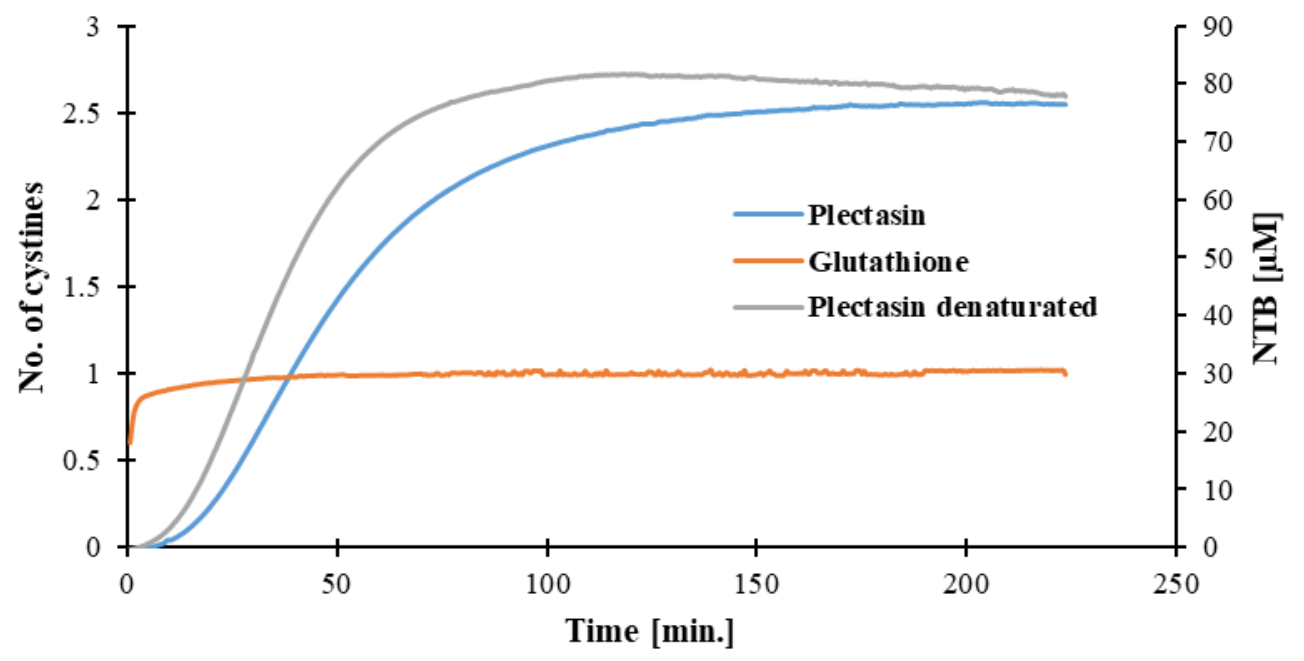

Figure 7: Kinetics of the disulfide bond breakage in native and denaturated Plectasin with Ellman reaction sulfite at $40^{\circ} \mathrm{C}$. The total number of disulfide bonds of the native and the denatured Plectasin shows a value of 2.6 with glutathione as a reference. The disulfide bridge in glutathione is almost instantaneously broken which is due to its high solvent exposure. In case of denatured versus native Plectasin, one can observe a difference in kinetics, and this is most likely due to differences in solvent accessibility where in the denatured state the disulfide bonds are highly exposed, resulting in faster kinetics. Both samples show a smooth transition from the bonded to the broken disulfide bridges.

\subsection{NMR study}

The unfolding of Plectasin upon gradual addition of TCEP to the final concentration of $80 \mathrm{mM}$ was followed by a series of $2 \mathrm{D}{ }^{1} \mathrm{H}-{ }^{13} \mathrm{C}$ HSQC, recorded after each addition of TCEP. Majority of peaks were in slow exchange, which means that during the course of titration the chemical shifts of folded peptide do not change in position, but rather slowly disappear. Therefore, the fraction of folded peptide can be monitored by measuring the intensity of peaks during titration. For all assigned Plectasin peaks, intensities were plotted against the concentration of added TCEP. 
Data, where the adjusted $\mathrm{R}^{2}$ values of the fit were less than 0.8 where discarded. For each amino acid, $\mathrm{EC}_{50}$ values of all peaks were averaged (Figure 8A) and mapped to the Plectasin structure (Figure 8B). Examining the normalized peaks intensities corresponding to individual cysteine residues suggest that the disulfide bond between 15CYS and 37CYS breaks first upon TCEP addition (Figure 9), with structural changes occurring only in the immediate neighbors as evident from chemical shift changes (Figure S5, S6). Based on $\mathrm{EC}_{50}$ values, the disulfide bond between 30CYS and 4CYS breaks next, followed by the disulfide bond between 39CYS and 19CYS. Only upon breakage of all three disulfide bonds does the structure completely unfold.

Upon addition of small concentrations of TCEP (up to $8 \mathrm{mM}$ ) to Plectasin, a decrease in 15CYS and 37CYS signal intensities are observed which are accompanied only by chemical shift perturbation of 36VAL $\mathrm{C}_{\alpha}$ and gradual decrease of intensity of their other neighboring amino acids. Lack of structural changes indicates that the native-like fold of Plectasin is retained even after the disulfide bond between 15CYS and 37CYS is broken. This complements the simulations results where no significant structural changes upon breakage of the 15CYS-37CYS bond as reflected by the low RMSD observed throughout the simulation (Figure 6A). At concentrations of TCEP higher than $20 \mathrm{mM}$, several new peaks with low intensity emerge in the HSQC spectra. The appearance of these new peaks correlates with reduction of the disulfide bond between 4CYS and 30CYS, however, the majority of Plectasin remains in the native-like fold as evident from ${ }^{1} \mathrm{H}$ and HSQC spectra. A number of new peaks that correspond to the Plectasin unfolded state and their intensity further increase when more TCEP is added and the fraction of the unfolded state increases. Complete unfolding of Plectasin is observed only after addition of $80 \mathrm{mM}$ TCEP, which is well beyond the concentration needed to reduce disulfide bonds. Therefore, one can conclude that all three disulfide bonds have to be reduced in order for Plectasin to completely unfold as also seen in MD simulations with all the three disulfide bridges broken (Figure 6B).
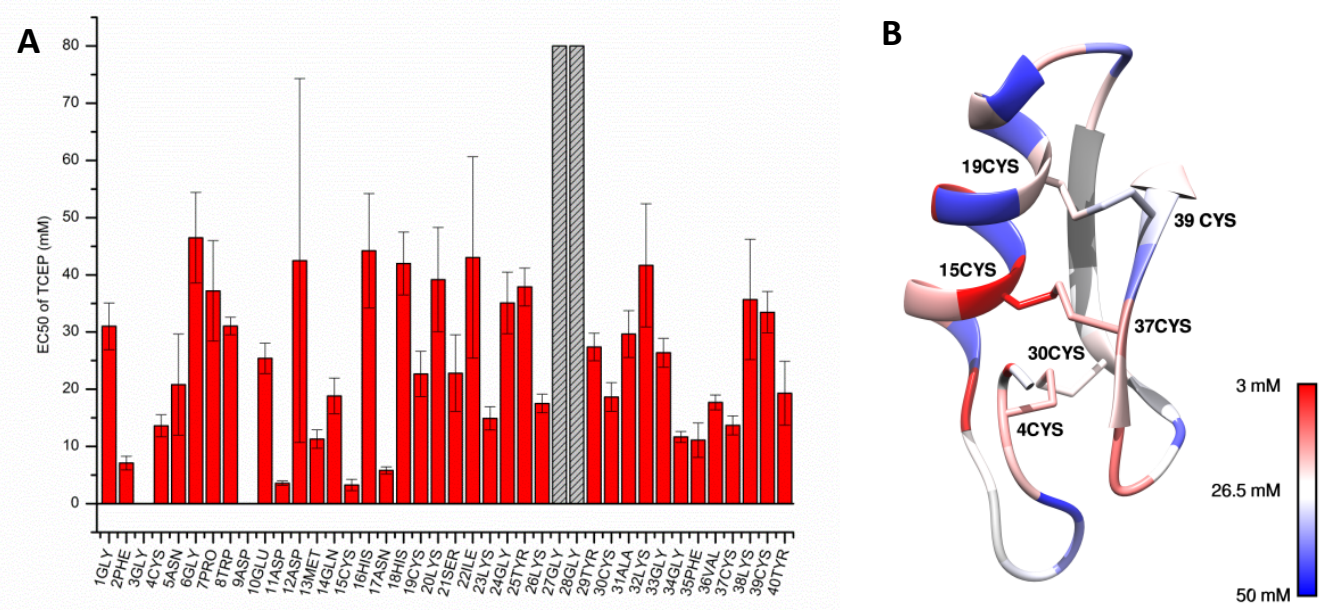

Figure 8: A) TCEP EC 50 values for individual amino acids in Plectasin. Data denoted in red were obtained by fitting signal intensities to a sigmoidal function. B) Structure of Plectasin (PDB ID: 3E7R ${ }^{8}$ ) colored by TCEP EC 50 value. 
For 27GLY and 28GLY peaks (denoted in grey), no changes in intensity were observed during the course of titration. For 3GLY and 9ASP, no reliable data were obtained due to low signal intensities.

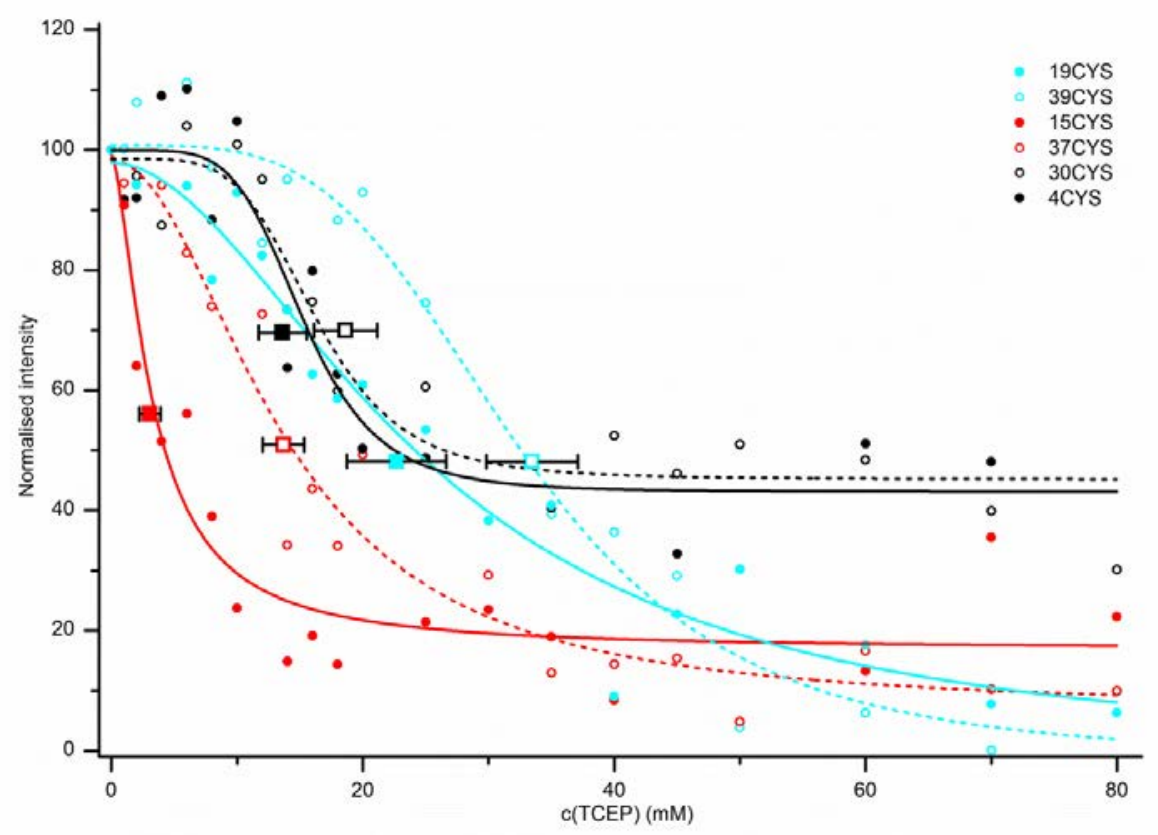

Figure 9: Plot of the normalized average peak intensity of cysteine pairs against the concentration of TCEP added, together with a fit to the sigmoidal function (lines) and $\mathrm{EC}_{50}$ values with erorrs (squares).The colors correspond to cysteine pairs involved in disulfide bonds.

\section{Conclusions}

Our computational study is the first reported study on the effect of $\mathrm{pH}$ and salt concentrations on the conformational stability of Plectasin and the three variants, thereof referred to PP40, PP41, PP42, and PP43. In this study, the working range for $\mathrm{pH}$ and salt concentrations were decided based on the conditions generally used to investigate the behaviour of potential protein/peptide biopharmaceuticals. The simulations predict that all Plectasin variants are conformationally stable at $\mathrm{pH}$. With an increase in $\mathrm{pH}$, native structures explore different conformers due to flexibility in the N-terminal loop containing the ASP-GLU-ASP-ASP stretch. Effect of different salt concentrations were studied by measuring the $\Gamma_{23}$. In general, $\mathrm{NaCl}$ has a high preference for PP40 as residue 9ASP is mutated in the other three variants. For all the Plectasin variants, sodium ions have higher tendency to adsorb to the peptide surface as compared to chloride ions. Equivalent $\Gamma_{23}$ values for PP40, PP41 and PP43 at $70 \mathrm{mM}$ and $140 \mathrm{mM} \mathrm{NaCl}$ imply that peptide surface is not saturated- However, for PP42, lower $\Gamma_{23}$ values are observed for $140 \mathrm{mM} \mathrm{NaCl}$ as compared to $70 \mathrm{mM} \mathrm{NaCl}$ indicate that surface saturation with $\mathrm{NaCl}$ has reached. Overall, the trend of $\Gamma_{23}$ for the two salt concentrations remains the same for all Plectasin variants, and the common residues interacting with the sodium ions are 10GLU, and 12ASP. At the different physiochemical conditions, peptide conformational stability is not affected significantly, which is in line with the fact that Plectasin has three disulfide bridges. The computational 
predictions of degree of involvement of three different disulfide bonds to the overall structural integrity of the protein was compared with experimental results. Quantitative assay were performed to study the time course of disulfide bond reduction. A lag phase with a single transition in the profile is observed which shows that it takes a relatively long time to break the first disulfide bond, and breaking of the first bond is enough to drive the reduction of the remaining two disulfide bonds. TI-MD simulations were performed to access the bond dissipation energy from each of the disulfide bonds. The first disulphide bridge to break is 15CYS-37CYS, which required 0.04 $\mathrm{Kcal} / \mathrm{mol}$ dissipation energy. This correlates well with classical all-atom MD simulations where low RMSD for the system with broken 15CYS-37CYS was observed indicating that structural integrity is maintained even though the cystine is reduced. TI-MD order of disulfide bond breaking is in good agreement with NMR, and the order of breaking is first 15CYS-37CYS, followed by 4CYS-30CYS, and last 19CYS-39CYS.

\section{Supporting information}

Figure S1, direction of motion of the Plectasin variants at pH 9; Figure S2, FEL displayed as a function of PC1 and PC2 for all Plectasin variants; Figure S3, profiles of derivates of the potential energy function $V(\lambda)$ with respect to $\lambda$; Figure S4, comparison of the kinetics of the disulfide bond breakage in native and denatured Plectasins at $25^{\circ} \mathrm{C}$ and $40^{\circ} \mathrm{C}$; Figure S5, overlap of ${ }^{1} \mathrm{H}-{ }^{13} \mathrm{C}$ NMR-HSQC spectra of Plectasin at different concentration of TCEP; Figure S6, ${ }^{1} \mathrm{H}$ spectra of Plectasin at different concentrations of TCEP.

\section{Author contribution}

\section{Corresponding authors}

E-mail: soemya@kemi.dtu.dk

E-mail: ghp@kemi.dtu.dk

\section{ORCID}

Sowmya Indrakumar: 0000-0002-5144-3261

Günther H.J. Peters: 0000-0001-9754-2663

\section{Author contributions}

S. I contributed with computational part, M. Z. contributed with NMR part, and C. P. contributed with quantitative disulfide reduction assay.

\section{Abbreviations}

RMSD: Root Mean Square Deviation

MD: Molecular Dynamics 
FEL: Free Energy Landscape

NMR: Nuclear Magnetic Resonance

PCA: Principal Component Analysis

TI-MD: Thermodynamic Integration Molecular Dynamics

IS: Ionic Strength

TCEP: tris(2-carboxyethyl)phosphine

NTSB: disodium 2-nitro-5-thiosulfobenzoat

NTB: 2-nitro-5-thiobenzoate

GnHCl: Guanidinium chloride

HSQC: Heteronuclear Single Quantum Coherence spectroscopy

TOCSY: Total Correlation Spectroscopy

PIC $\left(\Gamma_{23}\right)$ : Preferential Interaction Coefficient

SASA: Surface Accesibile Surface Area

\section{Acknowledgments}

This study was funded by a project part of the EU Horizon 2020 Research and Innovation program under the Marie Skłodowska-Curie grant agreement No 675074 - "Protein-excipient Interactions and Protein-Protein Interactions in formulation” (PIPPI); http://www.pippi.kemi.dtu.dk. Furthermore, we acknowledge VMD 1.9.3 ${ }^{24}$, pymol 1.8.4.2 ${ }^{41}$ and jupyter notebook plugins ${ }^{42}$. Simulations were performed on CPU/GPU cluster facilities at DTU Chemistry. NMR experiments were carried out at the NMR center in MIB, University of Manchester. Quantitative disulfide bond breaking assay were performed at Novozymes A/S.

\section{References}

(1) Lau, J. L.; Dunn, M. K. Therapeutic Peptides: Historical Perspectives, Current Development Trends, and Future Directions. Bioorganic Med. Chem. 2018. https://doi.org/10.1016/j.bmc.2017.06.052.

(2) Fosgerau, K.; Hoffmann, T. Peptide Therapeutics: Current Status and Future Directions. Drug Discovery Today. 2015. https://doi.org/10.1016/j.drudis.2014.10.003.

(3) Bak, A.; Leung, D.; Barrett, S. E.; Forster, S.; Minnihan, E. C.; Leithead, A. W.; Cunningham, J.; Toussaint, N.; Crocker, L. S. Physicochemical and Formulation Developability Assessment for Therapeutic Peptide Delivery-A Primer. AAPS J. 2015. https://doi.org/10.1208/s12248-014-9688-2. 
(4) Dror, R. O.; Jensen, M. Ø.; Borhani, D. W.; Shaw, D. E. Exploring Atomic Resolution Physiology on a Femtosecond to Millisecond Timescale Using Molecular Dynamics Simulations. J. Gen. Physiol. 2010. https://doi.org/10.1085/jgp.200910373.

(5) Mygind, P. H.; Fischer, R. L.; Schnorr, K. M.; Hansen, M. T.; Sönksen, C. P.; Ludvigsen, S.; Raventós, D.; Buskov, S.; Christensen, B.; De Maria, L.; et al. Plectasin Is a Peptide Antibiotic with Therapeutic Potential from a Saprophytic Fungus. Nature 2005, 437 (7061), 975-980. https://doi.org/10.1038/nature04051.

(6) Müller, A.; Klöckner, A.; Schneider, T. Targeting a Cell Wall Biosynthesis Hot Spot. Natural Product Reports. 2017. https://doi.org/10.1039/c7np00012j.

(7) Schneider, T.; Kruse, T.; Wimmer, R.; Wiedemann, I.; Sass, V.; Pag, U.; Jansen, A.; Nielsen, A. K.; Mygind, P. H.; Raventós, D. S.; et al. Plectasin, a Fungal Defensin, Targets the Bacterial Cell Wall Precursor Lipid II. Science (80-. ). 2010. https://doi.org/10.1126/science.1185723.

(8) Mandal, K.; Pentelute, B. L.; Tereshko, V.; Thammavongsa, V.; Schneewind, O.; Kossiakoff, A. A.; Kent, S. B. H. Racemic Crystallography of Synthetic Protein Enantiomers Used to Determine the X-Ray Structure of Plectasin by Direct Methods. Protein Sci. 2009, 18 (6), 1146-1154. https://doi.org/10.1002/pro.127.

(9) Li, Z.; Wang, X.; Wang, X.; Teng, D.; Mao, R.; Hao, Y.; Wang, J. Research Advances on Plectasin and Its Derivatives as New Potential Antimicrobial Candidates. Process Biochemistry. 2017. https://doi.org/10.1016/j.procbio.2017.02.006.

(10) Ahmad, S. Protein Oxidation an Overview of Metabolism of Sulphur Containing Amino Acid Cysteine. Front. Biosci. 2017. https://doi.org/10.2741/s474.

(11) Levine, R. L.; Mosoni, L.; Berlett, B. S.; Stadtman, E. R. Methionine Residues as Endogenous Antioxidants in Proteins. Proc. Natl. Acad. Sci. 1996. https://doi.org/10.1073/pnas.93.26.15036.

(12) Madhavi Sastry, G.; Adzhigirey, M.; Day, T.; Annabhimoju, R.; Sherman, W. Protein and Ligand Preparation: Parameters, Protocols, and Influence on Virtual Screening Enrichments. J. Comput. Aided. Mol. Des. 2013. https://doi.org/10.1007/s10822-013-9644-8.

(13) Gordon, J. C.; Myers, J. B.; Folta, T.; Shoja, V.; Heath, L. S.; Onufriev, A. H++: A Server for Estimating PKas and Adding Missing Hydrogens to Macromolecules. Nucleic Acids Res. 2005.

https://doi.org/10.1093/nar/gki464.

(14) Case, D. A.; Cheatham, T. E.; Darden, T.; Gohlke, H.; Luo, R.; Merz, K. M.; Onufriev, A.; Simmerling, C.; Wang, B.; Woods, R. J. The Amber Biomolecular Simulation Programs. Journal of Computational Chemistry. 2005. https://doi.org/10.1002/jcc.20290. 
(15) Mongan, J.; Case, D. A.; McCammon, J. A. Constant PH Molecular Dynamics in Generalized Born Implicit Solvent. J. Comput. Chem. 2004. https://doi.org/10.1002/jcc.20139.

(16) D.A. Case, R.M. Betz, W. Botello-Smith, D.S. Cerutti, T.E. Cheatham, III, T.A. Darden, R.E. Duke, T.J. Giese, H. Gohlke, A.W. Goetz, N. Homeyer, S. Izadi, P. Janowski, J. Kaus, A. Kovalenko, T.S. Lee, S. LeGrand, P. Li, C. Lin, T. Luchko, R. Luo, B. Madej, D. M. Y.; York, P. A. K. M.; Kollman, P. A. Amber 16. University of California, San Francisco. 2016. https://doi.org/10.1002/jcc.23031.

(17) Lindorff-Larsen, K.; Piana, S.; Palmo, K.; Maragakis, P.; Klepeis, J. L.; Dror, R. O.; Shaw, D. E. Improved Side-Chain Torsion Potentials for the Amber Ff99SB Protein Force Field. Proteins Struct. Funct.

Bioinforma. 2010. https://doi.org/10.1002/prot.22711.

(18) Mahoney, M. W.; Jorgensen, W. L. A Five-Site Model for Liquid Water and the Reproduction of the Density Anomaly by Rigid, Nonpolarizable Potential Functions. J. Chem. Phys. 2000. https://doi.org/10.1063/1.481505.

(19) Ryckaert, J. P.; Ciccotti, G.; Berendsen, H. J. C. Numerical Integration of the Cartesian Equations of Motion of a System with Constraints: Molecular Dynamics of n-Alkanes. J. Comput. Phys. 1977. https://doi.org/10.1016/0021-9991(77)90098-5.

(20) Darden, T.; York, D.; Pedersen, L. Particle Mesh Ewald: An N·log(N) Method for Ewald Sums in Large Systems. J. Chem. Phys. 1993. https://doi.org/10.1063/1.464397.

(21) Pastor, R. W.; Brooks, B. R.; Szabo, A. An Analysis of the Accuracy of Langevin and Molecular Dynamics Algorithms. Mol. Phys. 1988. https://doi.org/10.1080/00268978800101881.

(22) Berendsen, H. J. C.; Postma, J. P. M.; Van Gunsteren, W. F.; Dinola, A.; Haak, J. R. Molecular Dynamics with Coupling to an External Bath. J. Chem. Phys. 1984. https://doi.org/10.1063/1.448118.

(23) Roe, D. R.; Cheatham, T. E. PTRAJ and CPPTRAJ: Software for Processing and Analysis of Molecular Dynamics Trajectory Data. J. Chem. Theory Comput. 2013. https://doi.org/10.1021/ct400341p.

(24) Humphrey, W.; Dalke, A.; Schulten, K. VMD: Visual Molecular Dynamics. J. Mol. Graph. 1996. https://doi.org/10.1016/0263-7855(96)00018-5.

(25) Tan et al, S. Chap 8: Cluster Analysis: Basic Concepts and Algorithms. Introd. to Data Min. 2005. https://doi.org/10.1016/0022-4405(81)90007-8.

(26) Wolf, A.; Kirschner, K. N. Principal Component and Clustering Analysis on Molecular Dynamics Data of the Ribosomal L11·23S Subdomain. J. Mol. Model. 2013. https://doi.org/10.1007/s00894-012-1563-4. 
(27) Amadei, A.; Linssen, A. B. M.; Berendsen, H. J. C. Essential Dynamics of Proteins. Proteins Struct. Funct. Bioinforma. 1993. https://doi.org/10.1002/prot.340170408.

(28) David, C. C.; Jacobs, D. J. Principal Component Analysis: A Method for Determining the Essential Dynamics of Proteins. Methods Mol. Biol. 2014. https://doi.org/10.1007/978-1-62703-658-0_11.

(29) Brüschweiler, R. Collective Protein Dynamics and Nuclear Spin Relaxation. J. Chem. Phys. 1995. https://doi.org/10.1063/1.469213.

(30) Schellman, J. A. Fifty Years of Solvent Denaturation. Biophys. Chem. 2002. https://doi.org/10.1016/S03014622(02)00009-1.

(31) Shukla, D.; Shinde, C.; Trout, B. L. Molecular Computations of Preferential Interaction Coefficients of Proteins. J. Phys. Chem. B 2009. https://doi.org/10.1021/jp810949t.

(32) Fraczkiewicz, R.; Braun, W. Exact and Efficient Analytical Calculation of the Accessible Surface Areas and Their Gradients for Macromolecules. J. Comput. Chem. 1998. https://doi.org/10.1002/(SICI)1096987X(199802)19:3<319::AID-JCC6>3.0.CO;2-W.

(33) Thannhauser, T. W.; Konishi, Y.; Scheraga, H. A. Sensitive Quantitative Analysis of Disulfide Bonds in Polypeptides and Proteins. Anal. Biochem. 1984, 138 (1), 181-188. https://doi.org/10.1016/00032697(84)90786-3.

(34) Dolinsky, T. J.; Nielsen, J. E.; McCammon, J. A.; Baker, N. A. PDB2PQR: An Automated Pipeline for the Setup of Poisson-Boltzmann Electrostatics Calculations. Nucleic Acids Res. 2004.

https://doi.org/10.1093/nar/gkh381.

(35) Vrbka, L.; Jungwirth, P.; Bauduin, P.; Touraud, D.; Kunz, W. Specific Ion Effects at Protein Surfaces: A Molecular Dynamics Study of Bovine Pancreatic Trypsin Inhibitor and Horseradish Peroxidase in Selected Salt Solutions. Journal of Physical Chemistry B. 2006. https://doi.org/10.1021/jp0567624.

(36) Shirts, M. R.; Pitera, J. W.; Swope, W. C.; Pande, V. S. Extremely Precise Free Energy Calculations of Amino Acid Side Chain Analogs: Comparison of Common Molecular Mechanics Force Fields for Proteins. J. Chem. Phys. 2003. https://doi.org/10.1063/1.1587119.

(37) Simonson, T.; Carlsson, J.; Case, D. A. Proton Binding to Proteins: PKa Calculations with Explicit and Implicit Solvent Models. J. Am. Chem. Soc. 2004. https://doi.org/10.1021/ja039788m.

(38) Vaz, D. C. Enthalpic and Entropic Contributions Mediate the Role of Disulfide Bonds on the Conformational Stability of Interleukin-4. Protein Sci. 2005. https://doi.org/10.1110/ps.051593306. 
(39) Dagan, S.; Hagai, T.; Gavrilov, Y.; Kapon, R.; Levy, Y.; Reich, Z. Stabilization of a Protein Conferred by an Increase in Folded State Entropy. Proc. Natl. Acad. Sci. 2013. https://doi.org/10.1073/pnas.1302284110.

(40) Zhou, H. X. Loops, Linkages, Rings, Catenanes, Cages, and Crowders: Entropy-Based Strategies for Stabilizing Proteins. Acc. Chem. Res. 2004. https://doi.org/10.1163/15691497-12341418.

(41) DeLano, W. L. The PyMOL Molecular Graphics System, Version 1.8. Schrödinger LLC 2002. https://doi.org/citeulike-article-id:240061.

(42) Kluyver, T.; Ragan-Kelley, B.; Pérez, F.; Granger, B.; Bussonnier, M.; Frederic, J.; Kelley, K.; Hamrick, J.; Grout, J.; Corlay, S.; et al. Jupyter Notebooks - a Publishing Format for Reproducible Computational Workflows. In Positioning and Power in Academic Publishing: Players, Agents and Agendas; 2016. https://doi.org/10.3233/978-1-61499-649-1-87. 


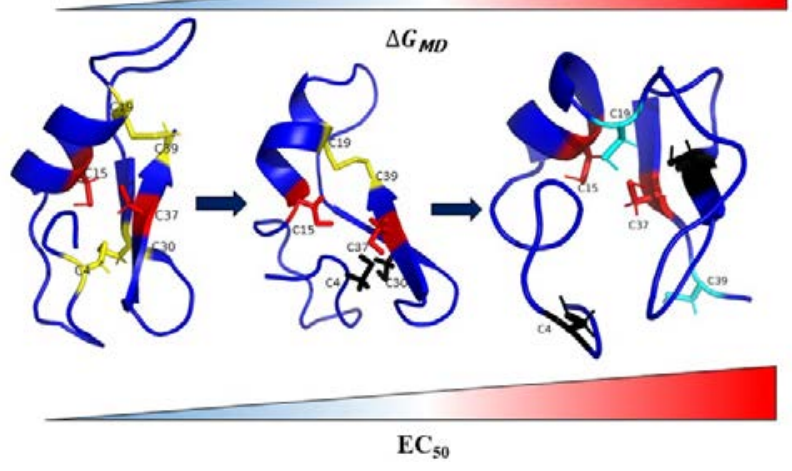

